\section{Successful Transvaginal Aspiration of Cervical Pregnancy after Failed Methotrexate Treatment: A Case Report}

\section{Abstract}

Background: Cervical pregnancies are infrequent and fatal. Systemic methotrexate (MTX) treatment is conventionally administered to preserve fertility and prevent hemorrhage. However, some patients do not respond to therapy.

Methods: Transvaginal aspiration because MTX treatment failed.

Results: Two cases were successfully treated by transvaginal aspiration. Serial ultrasound exams and blood beta-hCG confirmed resolution of cervical pregnancy.

Conclusion: Our results suggest that transvaginal aspiration should be considered an alternative upon failure of systemic MTX treatment or in the presence of contraindications for MTX injection.

Keywords: Cervical pregnancy; Transvaginal aspiration; Failed methotrexate treatment; Minimally invasive; In vitro fertilization; Methotrexate intolerance

Received: January 15, 2020; Accepted: January 30, 2020; Published: February 06, 2020

\section{Abbreviations}

IVF-ET: In vitro Fertilization Embryo Transfer; MTX: Methotrexate; BHCG: Beta Human Chorionic Gonadotropin

\section{Introduction}

Under normal physiologic conditions, a fertilized ovum migrates from the ampulla of the fallopian tube to implant on the wall of the endometrium. However, if the conception fails to reach its normal position within the endometrial cavity, an ectopic pregnancy occurs. Ectopic pregnancies result from extrauterine implantation of embryos and potentially result in fatal hemorrhage, thus warranting a prompt diagnosis and treatment. The cervix is a highly vascularized area, which is potentially suitable for the implantation of a fertilized ovum. However, it is also extremely vulnerable to heavy bleeding due to its suboptimal hemostatic mechanical capacity and its insusceptibility to respond to uterotonic agents. Most ectopic pregnancies occur in the Fallopian tubes, and less than $1 \%$ occur in the lining of the endocervical canal [1]. Commonly reported risk factors for cervical pregnancies include prior cesarean sections, dilation and curettage, and conception through in vitro fertilization (IVF) [2]. Cervical pregnancies occur in $0.1 \%$ of IVF-induced pregnancies

\author{
Fang $\mathbf{Y}^{1}$, Yu-Hung $\mathbf{L}^{1,2,3,4}$, \\ Jiann-Loung $\mathrm{H}^{1}$, \\ Lee-Wen $\mathrm{H}^{1,2,3}$, \\ Ting-Chi Huang ${ }^{1,2,3}$ and \\ Chun-Cheng Chu ${ }^{1 *}$
}

1 Department of Obstetrics and Gynecology, Shin Kong Wu Ho-Su Memorial Hospital, Shih Lin District, Taipei, Taiwan

2 School of Medicine, Fu-Jen Catholic University, New Taipei City, Taiwan

3 Department of Obstetrics and Gynecology, National Taiwan University Hospital, Zhongzheng District, Taipei, Taiwan

4 Department of Obstetrics and Gynecology, Taipei Medical University, Taipei, Taiwan

*Corresponding author: Chun-Cheng Chu

” ccchums1@yahoo.com.tw

Department of Obstetrics and Gynecology, Shin Kong Wu Ho-Su Memorial Hospital, No. 95, Wen Chang Road, Shih Lin District, Taipei, Taiwan.

Tel: 886228332211

Citation: Fang Y, Yu-Hung L, Jiann-Loung $\mathrm{H}$, Lee-Wen $\mathrm{H}$, Huang TC, et al. (2020) Successful Transvaginal Aspiration of Cervical Pregnancy after Failed Methotrexate Treatment: A Case Report. Vol.6 No.1:1

and account for $3.7 \%$ of cases of ectopic gestation resulting from IVF [3]. Patients with cervical pregnancies may present with uncontrollable and profuse vaginal bleeding, for which emergent hysterectomy may be life-saving. Different approaches have been implemented, but due to the rarity of this condition standard recommendations have not yet been elucidated. Recently, hysteroscopic resection of ectopic CP was described as an effective and fertility-preserving surgical therapy, having 
been used alone or in combination with another complementary therapy. Conservative management constitutes first-line treatment for individuals desiring future pregnancy. Herein, we present the cases of two patients with cervical pregnancy conceived through IVF. In both cases, treatment with transvaginal aspiration of the cervical gestational sacs was successful.

\section{Case Reports}

\section{Case 1}

A primiparous 38-year-old woman with cervical pregnancy induced through IVF-embryo transfer (IVF-ET) received multidose methotrexate (MTX); however, the gestational sac enlarged with an increase in $\beta$-human chorionic gonadotropin (hCG) levels, indicating treatment failure. Transvaginal aspiration was hence suggested. On the day of aspiration, the $\beta$-hCG level was 20585 $\mathrm{mIU} / \mathrm{mL}$, and fetal pole was observed (Figure 1). The gestational sac was undetectable upon sonography a week after aspiration and the $\beta$-hCG levels (determined serially) reverted to normalcy 2 months after the procedure.

\section{Case 2}

A primiparous 36-year-old woman with cervical pregnancy induced through IVF-ET (Figure 2) received systemic multidose MTX, which did not exert any therapeutic effects. On the day of aspiration, the $\beta$-hCG level was $8061.85 \mathrm{mlU} / \mathrm{mL}$. Two weeks after aspiration, her $\beta$-hCG level was undetectable.

\section{Discussion}

The incidence of cervical pregnancies, unlike that of tubal pregnancies, is extremely low; hence, no proven guidelines for pharmacotherapeutic or surgical treatment have been established. Before 1980, cervical pregnancies were diagnosed when dilation and curettage for presumed incomplete abortions resulted in unexpected hemorrhage [4]. Delayed diagnosis and treatment potentially result in massive bleeding, warranting hysterectomy. Advancements in ultrasound imaging have helped experienced physicians to identify such patients as early as the

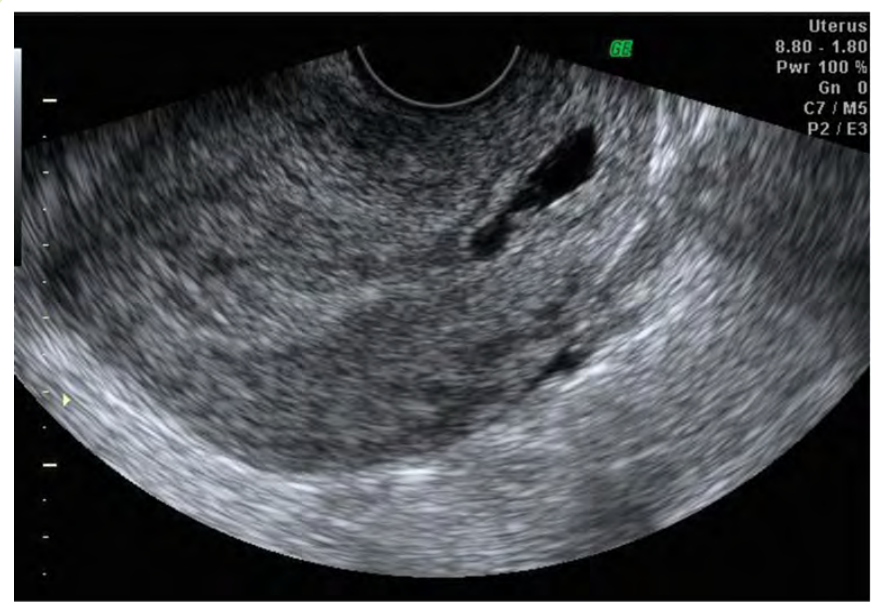

Figure 1 Transvaginal ultrasound image of Case 1.

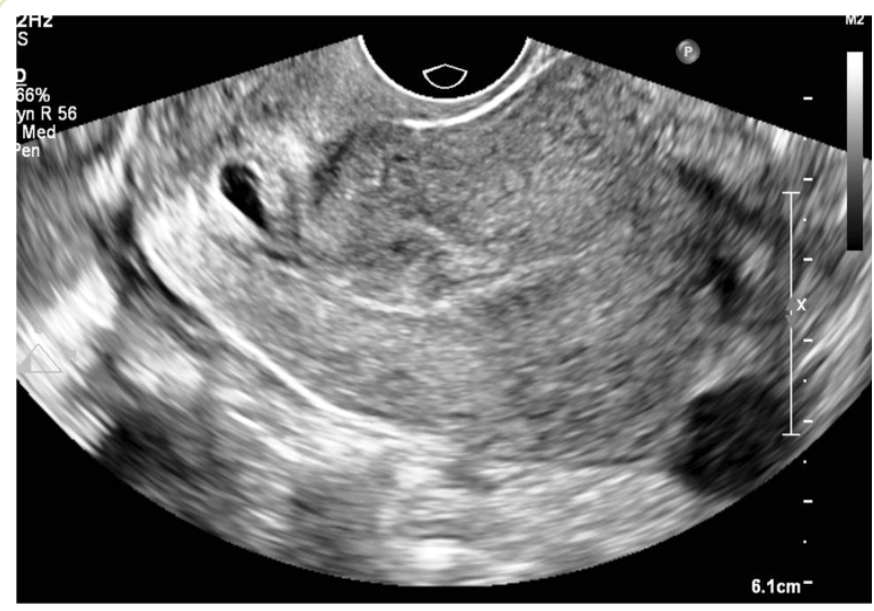

Figure 2 Transvaginal ultrasound image of Case 2.

fifth gestational week.

For hemodynamically stable patients receiving an early diagnosis, conservative and fertility-sparing treatment is administered. Scholars have proposed systemic MTX treatment alone or intraamniotic MTX administration followed by ultrasound-guided fetal intracardiac injection of potassium chloride and mifepristone, uterine artery embolization, and dilation and curettage for clinically managing cervical pregnancy [5-11]. However, patients experiencing treatment failure with acute hemorrhage have also been reported $[12,13]$. The primary risk for curettage is severe hemorrhage, which predisposes to cervical implantation in subsequent pregnancy $[2,14]$. Uterine artery embolization may control heavy bleeding; however, subsequent fertility and pregnancy outcomes are not adequately promising [15].

In Case 1 described herein, despite systemic multidose MTX administration, an increase in the patient's $\beta$-hCG level confirmed treatment failure. To spare fertility and prevent massive bleeding, salvage therapy was administered through transvaginal aspiration with the patient's consent. Based on the favorable response observed in Case 1, the same procedure was performed in Case 2, wherein the patient's condition was diagnosed at an even earlier gestational stage, thus preventing the increased risk of treatment failure. Bleeding levels were minimal in both cases, with no need for hospitalization.

\section{Conclusion}

The overall rate of ectopic pregnancy continues to rise. Early recognition of the signs and symptoms of ectopic pregnancy is paramount to achieving positive patient outcomes. Ectopic pregnancy remains challenging despite the substantial increase in number of incidences. Cervical pregnancies are rare but potentially lethal, with an increasing incidence owing to artificial reproductive methods. Thus far, no consensus has been obtained regarding the management of this condition. Our results suggest that transvaginal aspiration should be considered an alternative upon failure of systemic MTX treatment or in the presence of contraindications for MTX injection. A prompt diagnosis of 
cervical pregnancy would guarantee the success of conservative treatment.

\section{Acknowledgement}

None.

\section{Author's contributions}

C.-C. C., L.-W. Huang, and J.-L. Hwang analyzed patient data. I-F. Y. and Y.-H. L. performed the procedure. T. C. H. drafts the paper. All authors participated in patient care, read and approved the manuscript.

\section{References}

1 Bouyer J, Coste J, Fernandez H, Pouly JL, Job-Spira N (2002) Sites of ectopic pregnancy: A 10 year population-based study of 1800 cases. Hum Reprod 17: 3224.

2 Sharma A, Ojha R, Mondal S, Chattopadhyay S, Sengupta P (2013) Cervical intramural pregnancy: A report of a rare case. Niger Med J 54: 271-273.

3 Karande VC, Flood JT, Heard N, Veeck L, Muasher SJ (1991) Analysis of ectopic pregnancies resulting from in-vitro fertilization and embryo transfer. Hum Reprod 6: 446

4 Leeman LM, Wendland CL (2000) Cervical ectopic pregnancy. Arch Fam Med 9: 72-77.

5 Verma U, Goharkhay N (2009) Conservative management of cervical ectopic pregnancy. Fertil Steril 91: 671-674.

6 Kirk E, Condous G, Haider Z, Syed A, Ojha K, et al. (2006) The conservative management of cervical ectopic pregnancies. Ultrasound Obstet Gynecol 27: 430.

7 Mesogitis S, Pilalis A, Daskalakis G, Papantoniou N, Antsaklis A (2005) Management of early viable cervical pregnancy. BJOG 112:409.

8 Mitra AG, Harris-Owens M (2000) Conservative medical management of advanced cervical ectopic pregnancies. Obstet Gynecol Surv 55: 385.

\section{Informed Consent}

Patients' consent form were obtained before procedure and held by hospital documentation team.

\section{Conflict of Interest}

The authors declare that they have no conflicts of interest.

\section{Funding}

This work was supported by Shin Kong Hospital.

9 Weibel HS, Alserri A, Reinhold C, Tulandi T (2012) Multidose methotrexate treatment of cervical pregnancy. J Obstet Gynaecol Can 34: 359-362.

10 Shrestha E, Yang Y, Li X, Zhang Y (2011) Successful conservative management with methotrexate and mifepristone of cervical pregnancy. J Biomed Res 25: 71-73.

11 Adabi K, Nekuie S, Rezaeei Z, Rahimi-Sharbaf F, Banifatemi S, et al. (2013) Conservative management of cervical ectopic pregnancy: systemic methotrexate followed by curettage. Arch Gynecol Obstet 288: 687-689.

12 Mangino FP, Ceccarello M, Di Lorenzo G, D'Ottavio G, Bogatti P, et al. (2014) Successful rescue hysteroscopic resection of a cervical ectopic pregnancy previously treated with methotrexate with no combined safety precautions. Clin Exp Obstet Gynecol 41: 214-216.

13 Pereira N, Grias I, Foster SE, Della Badia CR (2013) Acute hemorrhage related to a residual cervical pregnancy: management with curettage, tamponade, and cerclage. J Minim Invasive Gynecol 20: 907-911.

14 Kim TJ, Seong SJ, Lee KJ, Lee JH, Shin JS, et al. (2004) Clinical outcomes of patients treated for cervical pregnancy with or without methotrexate. J Korean Med Sci 19: 848.

15 Takeda J, Makino S, Ota A, Tawada T, Mitsuhashi N, et al. (2014) Spontaneous uterine rupture at 32 weeks of gestation after previous uterine artery embolization. J Obstet Gynaecol Res 40: 243. 